Gravitation 83 Cosmology, Vol. 6 (2000), No. 4 (24), pp. 1-10

(C) 2000 Russian Gravitational Society

\title{
HOLOGRAPHIC ENTROPY ON THE BRANE FROM DS/CFT CORRESPONDENCE
}

\author{
Sachiko Ogushid \\ Yukawa Institute for Theoretical Physics, Kyoto University, Kyoto 606-8502, JAPAN
}

\begin{abstract}
We discuss the relationship between the entropy of de Sitter (dS) Schwarzschild space and that of the CFT which lives on the brane by using Friedmann-Robertson-Walker (FRW) equations and Cardy-Verlinde formula. The cosmological constant appears on the brane with time-like metric in dS Schwarzschild background. On the other hand, in case of the brane with space-like metric in dS Schwarzschild background, the cosmological constant of the brane does not appear because we can choose brane tension to cancel it. We show that when the time-like brane crosses the black hole horizon of dS Schwarzschild black hole, the entropy of the CFT exactly agrees with the black hole entropy of 5-dimensional dS background. This report is based on the work [1]].
\end{abstract}

The holographic duality which connects $d+1-$ dimensional gravity in Anti-de Sitter (AdS) background with $d$-dimensional conformal field theory (CFT) has been discussed vigorously for some years. The one of the evidences for the existence of the AdS/CFT correspondence is that the isometry of $d+1$-dimensional AdS space $S O(d, 2)$ is identical with the conformal symmetry of $d$-dimensional Minkowski space. Recently much attention has been paid for the duality between de Sitter (dS) gravity and CFT by the analogy of the AdS/CFT correspondence, which is called dS/CFT correspondence, because the isometry of $d+1$-dimensional de Sitter space, $S O(d+1,1)$, exactly agrees with the conformal symmetry of $d$-dimensional Euclidean space. Thus it might be natural to expect the correspondence between $d+1$-dimensional gravity in de Sitter space and $d$-dimensional Euclidean CFT. The dS/CFT correspondence is initiated by C.M. Hull [2], A. Strominger [3] and E. Witten [4]. Following their works, much attention has been paid for dS/CFT correspondence [- [1], [5], [6].

Moreover the holographic principle between the radiation dominated Friedmann-Robertson-Walker (FRW) universe in $d$-dimensions and same dimensional CFT with a dual $d+1$-dimensional AdS description was studied by E. Verlinde [7]. Especially, we can see the correspondence between black hole entropy and the entropy of the CFT which is derived by making the appropriate identifications for FRW equation with the generalized Cardy formula. The Cardy formula is originally the entropy formula of the CFT only for 2-dimensions [8], while the generalized Cardy formula expresses that of the CFT for any dimensions [7]. From the point of brane-world physics [9], the CFT/FRW relation sheds further light on the study of the brane CFT in the background of AdS Schwarzschild black hole [10]. There was much activity on the studies of related questions [5], 11], 12], 113], 14].

\footnotetext{
${ }^{1}$ e-mail: ogushi@yukawa.kyoto-u.ac.jp
}

The purpose of this report is the further study of the CFT in de Sitter (dS) Schwarzschild background guided by the analogy of AdS Schwarzschild background. The investigation of dS brane in dS Schwarzschild background in terms of FRW equations has been initiated in the work 12. The important difference between AdS space and $\mathrm{dS}$ space is the sign of cosmological constant. In case of the brane with time-like (Minkowski) metric on AdS Schwarzschild background [10], the cosmological constant does not appear because we can choose brane tension to cancel it. But it is impossible for dS Schwarzschild background with the positive cosmological constant. We will see that the cosmological constant always appears in FRW equations deduced from timelike brane trajectory in dS Schwarzschild background 1 . It is interesting to note that the brane with space-like (Euclidean) metric in dS Schwarzschild background, the cosmological constant of the brane does not appear for the same reason in case of AdS Schwarzschild background. From the point of view of the dS/CFT correspondence, the investigation of space-like brane is more interesting than that of time-like brane.

Furthermore we argue the entropy of the brane CFT which is derived by using generalized Cardy formula for both time-like and space-like branes. We will see that when the time-like brane crosses the black hole horizon of dS Schwarzschild black hole, the CFT is identical with the black hole entropy of 5 -dimensional dS background as it happens in the AdS/CFT correspondence.

\section{FRW equations in the background of de Sitter Schwarzschild black hole}

We first consider a 4-dimensional time-like brane in 5dimensional dS Schwarzschild background. From the

\footnotetext{
${ }^{1}$ The systematic method deriving brane cosmological equation for a brane embedded in a bulk with a cosmological constant has been first examined by the works 13 .
} 
analogy of the AdS/CFT correspondence, we can regard that 4-dimensional CFT exists on the brane which is the boundary of the 5-dimensional dS Schwarzschild background. The dynamics of the brane is described by the boundary action:

$$
\mathcal{L}_{b}=\frac{-1}{8 \pi G_{5}} \int_{\partial \mathcal{M}} \sqrt{-g} \mathcal{K}+\frac{\kappa}{8 \pi G_{5}} \int_{\partial \mathcal{M}} \sqrt{-g} .
$$

Here $G_{5}$ is 5 -dimensional bulk Newton constant, $\partial \mathcal{M}$ denotes the surface of the brane, $g$ is the determinant of the induced metric on $\partial \mathcal{M}, \mathcal{K}_{i j}$ is the extrinsic curvature, and $\mathcal{K}=\mathcal{K}_{i}^{i}, \kappa$ is a parameter related to tension of the brane.

From this Lagrangian, we can get the equation of motion of the brane as 10]:

$$
\mathcal{K}_{i j}=\frac{\kappa}{2} g_{i j},
$$

which implies that $\partial \mathcal{M}$ is a brane of constant extrinsic curvature. The bulk action is given by 5 -dimensional Einstein action with cosmological constant. The dS Schwarzschild space is one of the exact solutions of bulk equations of motion and can be written in the following form,

$$
\begin{aligned}
d s_{5}^{2} & =\hat{G}_{\mu \nu} d x^{\mu} d x^{\nu} \\
& =-\mathrm{e}^{2 \rho} d t^{2}+\mathrm{e}^{-2 \rho} d r^{2}+r^{2} d \Omega_{3}^{2}, \\
\mathrm{e}^{2 \rho} & =\frac{1}{r^{2}}\left(-\mu+r^{2}-\frac{r^{4}}{l^{2}}\right) .
\end{aligned}
$$

Here $l$ is the curvature radius of dS and $\mu$ is the black hole mass. In case of AdS Schwarzschild gravity, there is a holographic relation between FRW brane universe which is reduction from AdS Schwarzschild background and boundary CFT which lives on the brane [10], 111. We assume that there are some holographic relations between FRW universe which is reduction from dS Schwarzschild background and boundary CFT. To investigate it, we rewrite dS Schwarzschild metric (3) in the form of FRW metric by using a new time parameter $\tau$ following the method of the work [10]. And the parameter $t$ and $r$ in (3) are the function of $\tau$, namely $r=r(\tau), t=t(\tau)$. For the purpose of getting the 4-dimensional FRW metric, we impose the following condition,

$$
-e^{2 \rho}\left(\frac{\partial t}{\partial \tau}\right)^{2}+e^{-2 \rho}\left(\frac{\partial r}{\partial \tau}\right)^{2}=-1 .
$$

Thus we obtain FRW metric:

$$
d s_{4}^{2}=g_{i j} d x^{i} d x^{j}=-d \tau^{2}+r^{2} d \Omega_{3}^{2} .
$$

The extrinsic curvature, $\mathcal{K}_{i j}$, of the brane can be calculated and expressed in term of the function $r(\tau)$ and $t(\tau)$. Thus one rewrites the equations of motion (2) as

$$
\frac{d t}{d \tau}=-\frac{\kappa r}{2} e^{-2 \rho} .
$$

Using (4) and (6), we can derive FRW equation for a radiation dominated universe, Hubble parameter $H$ which is defined by $H=\frac{1}{r} \frac{d r}{d \tau}$ is given by

$$
H^{2}=\frac{1}{l^{2}}-\frac{1}{r^{2}}+\frac{\mu}{r^{4}}+\frac{\kappa^{2}}{4} .
$$

Following AdS Schwarzschild gravity case 10, we choose $\kappa=2 / l$ from now on 2 . This equation can be rewritten by using 4-dimensional energy $E_{4}$ and volume $V$ in the form of the standard FRW equation with the positive cosmological constant $\Lambda$ :

$$
\begin{aligned}
H^{2} & =-\frac{1}{r^{2}}+\frac{8 \pi G_{4}}{3} \frac{E_{4}}{V}+\frac{\Lambda}{3}, \\
E_{4} & =\frac{3 \mu V}{8 \pi G_{4} r^{4}}, \quad \Lambda=\frac{6}{l^{2}} .
\end{aligned}
$$

Here $G_{4}$ is the 4-dimensional gravitational coupling, which is defined by

$$
G_{4}=\frac{2 G_{5}}{l} .
$$

$E_{4}$ can be regarded as 4-dimensional energy on the brane in dS Schwarzschild background which is identical with AdS Schwarzschild case. The cosmological constant $\Lambda$ does not appear in AdS Schwarzschild background because we can choose brane tension $\kappa$ to cancel the cosmological constant of AdS Schwarzschild background. But it is impossible for dS Schwarzschild case because if we choose brane tension to cancel the cosmological constant of dS Schwarzschild background, the brane tension should be imaginary.

By differentiating eq.(8) with respect to $\tau$, we obtain the second FRW equation:

$$
\begin{aligned}
\dot{H} & =-4 \pi G_{4}\left(\frac{E_{4}}{V}+p\right)+\frac{1}{r^{2}}, \\
p & =\frac{\mu}{8 \pi G_{4} r^{4}} .
\end{aligned}
$$

Here $p$ is 4-dimensional pressure of the matter on the boundary. From eqs.(8) and (10), we find that the energy-momentum tensor is traceless:

$$
T^{\text {matter } \mu_{\mu}}=-\frac{E_{4}}{V}+3 p=0 .
$$

Therefore the matter on the brane can be regarded as the radiation, which is consistent with the work [6] whose calculation has been done in some asymptotically dS space in a sense of dS/CFT correspondence. This result means the field theory on the brane should be CFT as in case of AdS Schwarzschild background [10].

Next, we consider space-like brane in 5-dimensional dS Schwarzschild background. Similarly, we impose the

\footnotetext{
${ }^{2}$ From the point of view of brane-world physics [9], the tension of brane should be determined without ambiguity. In fact, we can calculate it to cancel the leading divergence of bulk AdS Schwarzschild 11.
} 
following condition to obtain space-like brane metric instead of eq.(4):

$$
-e^{2 \rho}\left(\frac{\partial t}{\partial \tau}\right)^{2}+e^{-2 \rho}\left(\frac{\partial r}{\partial \tau}\right)^{2}=1
$$

Thus we get following FRW-like metric:

$$
d s_{4}^{2}=g_{i j} d x^{i} d x^{j}=d \tau^{2}+r^{2} d \Omega_{3}^{2} .
$$

Note that this metric is also derived by Wick-rotation $\tau \rightarrow i \tau$ in eq.(5). We again calculate the equations of motion and the extrinsic curvature of space-like brane instead of (2) and (6. These equations lead FRW like equation as follows: 3

$$
H^{2}=-\frac{1}{l^{2}}+\frac{1}{r^{2}}-\frac{\mu}{r^{4}}+\frac{\kappa^{2}}{4} .
$$

To cancel the cosmological constant, we take $\kappa=2 / l$ in the same way of AdS Schwarzschild gravity [10]. We assume this equation can be rewritten by using 4 dimensional energy $E_{4}$ and volume $V$ by the analogous form of the standard FRW equations:

$$
\begin{aligned}
H^{2} & =\frac{1}{r^{2}}-\frac{8 \pi G_{4}}{3} \frac{E_{4}}{V}, \quad E_{4}=\frac{3 \mu V}{8 \pi G_{4} r^{4}} . \\
\dot{H} & =4 \pi G_{4}\left(\frac{E_{4}}{V}+p\right)-\frac{1}{r^{2}}, \quad p=\frac{\mu}{8 \pi G_{4} r^{4}} .
\end{aligned}
$$

The reason why the sign of FRW equations is different from the standard FRW equations (8) results from the condition (12), namely $\tau \rightarrow i \tau$ in eq.(5). From eqs. (15) and (16), we find the energy-momentum tensor is traceless again.

We stress again that we can take cosmological constant to zero for FRW-like equation in space-like brane in $d S$ Schwarzschild background as the same way in the AdS/CFT correspondence. This will imply that the dS/CFT correspondence can be valid for space-like brane in dS Schwarzschild background.

\section{The Cardy-Verlinde formula for the dS/CFT correspondence}

E. Verlinde showed that the $d$-dimensional FRW equation can be regarded as an analogue of the Cardy formula of 2-dimensional CFT [7].

$$
S_{4}=2 \pi \sqrt{\frac{c}{6}\left(L_{0}-\frac{c}{24}\right)} .
$$

For time-like brane of 5-dimensional dS Schwarzschild background, identifying

$$
\begin{aligned}
\frac{2 \pi}{3}\left(E_{4} r+\frac{\Lambda V r}{8 \pi G_{4}}\right) & \Rightarrow 2 \pi L_{0}, \\
\frac{V}{8 \pi G_{4} r} & \Rightarrow \frac{c}{24}, \\
\frac{H V}{2 G_{4}} & \Rightarrow S_{4},
\end{aligned}
$$

\footnotetext{
${ }^{3}$ In the works [13], 14, the similar equations to eqs.(7), (14) were obtained in terms of Ricci scalar of the induced metric of the brane.
}

FRW equation (8) has the form (17). The effect of the cosmological constant appears in Cardy formula. We included contribution of the cosmological constant in $L_{0}$ because it shifts the vacuum energy. This means the cosmological entropy bound [7] should be changed. The Bekenstein bound [7] in 4-dimensions is

$$
S \leq S_{B}, \quad S_{B} \equiv \frac{2 \pi}{3} E r .
$$

Using eq. (18), the Bekenstein entropy bound should be changed as follows:

$$
S \leq S_{B}, \quad S_{B} \equiv \frac{2 \pi}{3}\left(E r+\frac{\Lambda V r}{8 \pi G_{4}}\right) .
$$

Then we find out that the effect of the cosmological constant appears in the change of the Bekenstein entropy bound.

For the case of space-like brane, identifying

$$
\begin{aligned}
\frac{2 \pi}{3} E_{4} r & \Rightarrow 2 \pi L_{0}, \\
\frac{V}{8 \pi G_{4} r} & \Rightarrow \frac{c}{24}, \\
-i \frac{H V}{2 G_{4}} & \Rightarrow S_{4},
\end{aligned}
$$

Here $H$ changes as $H \rightarrow-i H$ since $H$ is defined by $H=\frac{1}{r} \frac{d r}{d \tau}$ and $\frac{d}{d \tau}$ change as $-i \frac{d}{d \tau}$ by the Wick-rotation, $\tau \rightarrow i \tau$. The third correspondence in eq.(21) is identical with space-like brane in AdS Schwarzschild background [5] exactly.

The moment when time-like brane crosses the black hole horizont $r=r_{H}$ which is derived from $e^{2 \rho\left(r_{H}\right)}=0$, the Hubble parameter in (7) becomes as $H= \pm 1 / l$. Here the plus sign corresponds to the expanding brane universe and the minus one to the contracting universe. We choose the expanding case. Note that the relation $H= \pm 1 / l$ is the same form as the case of $\operatorname{AdS}$ Schwarzschild black hole [10, (11]. Using eqs.(9), (18), we obtain 4-dimensional entropy $S_{4}$ as follows:

$$
S_{4}=\frac{V}{2 l G_{4}}=\frac{V}{4 G_{5}} .
$$

This entropy is nothing but the Bekenstein-Hawking entropy of 5-dimensional dS black hole similar to AdS/CFT correspondence [10], [11].

We now understand that discovered relation between FRW equations and entropy formulas [7] can be also applied to dS Schwarzschild background. If we take timelike brane which is the boundary of dS Schwarzschild background, the cosmological constant of the brane appears in FRW equations. Therefore the effect of cosmological constant contributes to raising the the Bekenstein entropy bound. But if we take space-like brane in dS Schwarzschild background, we obtain the approximately same result of AdS Schwarzschild black hole [10], 11]. The difference between space-like brane in dS

\footnotetext{
${ }^{4}$ The time-like brane can only cross the black hole horizon
} 
Schwarzschild background and time-like brane in AdS Schwarzschild background is the sign of FRW equations and third correspondence in eq.(21). When the time-like brane crosses the black hole horizon of dS Schwarzschild black hole, the entropy formula of the CFT exactly agrees with the black hole entropy of 5-dimensional dS background as it happens in the AdS/CFT correspondence. This implies that the holographic principle holds true for dS Schwarzschild background.

\section{Acknowledgement}

I would like to thank S. Nojiri, S.D. Odintsov for useful discussions. I wish to thank the conference organizers for their hospitality. This work is supported in part by the Japan Society for the Promotion of Science under the Postdoctoral Research Program.

\section{References}

[1] S. Ogushi, Mod. Phys. Lett. A17 (2002) 51, hepth/0111008.

[2] C.M. Hull, JHEP 9807(1998) 021, hep-th/9806146.

[3] A. Strominger, JHEP 0110(2001) 034, hep-th/0106113.

[4] E. Witten, hep-th/0106109.

[5] S. Nojiri, S.D. Odintsov and S. Ogushi, hep-th/0205187 to appear in Int. J. Mod. Phys. Further references are contained therein.

[6] R.-G. Cai, Y.S. Myung and Y.-Z. Zhang, Phys. Rev. D65, 084019 (2002), hep-th/0110234.

[7] E. Verlinde, hep-th/0008140.

[8] J.L. Cardy, Nucl. Phys. B270 (1986) 967.

[9] L. Randall and R. Sundrum, Phys. Rev. Lett. 83 (1999) 3370, hep-th/9905221; Phys. Rev. Lett. 83 (1999) 4690, hep-th/9906064.

[10] I. Savonije and E. Verlinde, Phys. Lett. B 507 (2001) 305, hep-th/0102042.

[11] S. Nojiri, S.D. Odintsov and S. Ogushi, Int. J. Mod. Phys. A16(2001) 5085, hep-th/0105117.

[12] S. Nojiri, S.D. Odintsov, JHEP 0112(2001) 033, hepth/0107134.

[13] P. Binetruy, C. Deffayet and D. Langlois, Nucl. Phys. B565(2000) 269, hep-th/9905012.

[14] P. Binetruy, C. Deffayet, U. Ellwanger and D. Langlois, Phys. Lett.B477(2000) 285, hep-th/9910219, Further references are contained therein. 